\title{
Superficial and profound considerations about sperm quality
}

\section{Pierre Comizzoli ${ }^{1}$}

Received: 7 June 2019 / Accepted: 11 June 2019 / Published online: 25 June 2019

(C) This is a U.S. Government work and not under copyright protection in the US; foreign copyright protection may apply 2019

Sperm quality is defined by the ability to reach and bind to an egg, fertilize it, and sustain an activated (then successful) embryonic development program. These attributes are valid for in vivo as well as in vitro conditions (IVF). Sperm quality is determined by multiple properties and their constitutive components (from the surface to the core of the cell) ultimately ensuring delivery of an intact paternal genome in a timely fashion. In addition to fertilization success, it is now clear that paternal factors have long-lasting effects on embryo development and offspring health [1]. To date, studies on the developmental competence of mammalian gametes are far more numerous for oocytes than those dedicated to the characterization of the sperm competence. This issue of JARG begins to fill the relative void regarding the male gamete with three articles providing new insights about human sperm quality, including some traits that could be used as predictors of pregnancy success.

In terms of superficial components, the quality of the sperm membrane is tightly associated with normal semen analysis and ability to fertilize an oocyte. The article by Craig et al. [2] demonstrates that the lipid composition of the sperm membrane is essential to the sperm structure and function. In this study, ejaculates from 70 men underwent a complete semen analysis (including volume, count, motility, progression, agglutination, viscosity, morphology, and pH). Lipid membranes then were extracted from the cells and analyzed by gas chromatography. For the first time, a correlation is made between heathy human spermatozoa and the presence of very long chain of polyunsaturated fatty acids (VLC-PUFA). Authors show a positive correlation between the levels of VLCPUFA with sperm count and total motile count. Interestingly, low VLC-PUFA levels do not result from inadequate PUFA precursors in metabolism, which means that fertility could be restored through VLC-PUFA supplementation in diet.

Pierre Comizzoli

comizzolip@si.edu

1 Smithsonian Conservation Biology Institute, National Zoological Park, Washington, DC, USA
Besides potential issues related to membrane composition, sperm binding failure and absence of fertilization are a major limitation of human IVF. In a new study conducted by Zhao and Kan [3], this particular problem is addressed through a series of experiments involving oviductin - an oviductal protein previously shown to play a key role in the fertilization process. Using a recombinant human oviductin (rHuOVGP1) produced in their laboratory, the group collected data suggesting that rHuOVP1 enhances sperm capacitation, in part, through the increase in the level of tyrosine phosphorylation of sperm proteins that are associated with another protein located in fibrous sheath of the sperm tail (AKAP3). They clearly showed that rHuOVGP1 binds to the zona pellucida of human oocytes and even increases the incidence of spermzona binding. Thus, supplementing the sperm capacitating medium with rHuOVGP1 should be particularly useful for enhancement of the IVF success.

Lastly, the study from Bichara et al. [4] sheds new light on deeper aspects, such as the impact of sperm nuclear damage on ICSI outcomes. To better characterize this negative effect, the authors examined clinical pregnancy rates after ICSI according to several types of sperm DNA damage (sperm head vacuoles, chromatin condensation, DNA fragmentation, and aneuploidy) that were explored simultaneously in 132 couples with male factor or mixed infertility. The study specifically highlights the value of sperm chromatin condensation defects (simply identified by Aniline Blue staining) as a reliable indicator for pregnancy outcomes after ICSI. Sperm chromatin condensation defects are more frequently observed in couples with ICSI failure and should be considered a negative predictive factor for the occurrence of clinical pregnancy. The authors also emphasize that developing novel assessment approaches for sperm chromatin quality, including sperm nucleoprotein composition, and its impact on ICSI outcomes are an important next step.

In sum, advances in the study of sperm quality will increasingly depend on multi-prong strategies taking into consideration gametic properties from the superficial to the deep components - the latter is especially notable given mounting interest in the role of epigenetics modifications. While we 
need more knowledge about aspects of sperm quality at all levels, these three articles also show that it is fundamental and possible to develop in parallel approaches to mitigate issues and restore sperm functions.

\section{References}

1. Colaco S, Sakkas D. Paternal factors contributing to embryo quality. J Assist Reprod Genet. Springer US; 2018 [cited 2019 May 24];35: 1953-68. Available from: http://link.springer.com/10.1007/s10815018-1304-4

2. Craig LB, Brush RS, Sullivan MT, Zavy MT, Agbaga M-P, Anderson RE. Decreased very long chain polyunsaturated fatty acids in sperm correlates with sperm quantity and quality. J Assist Reprod Genet. Springer US; 2019 [cited 2019 May 24];1-7. Available from: http://link.springer.com/10.1007/s10815-019-01464-3
3. Bichara C, Berby B, Rives A, Jumeau F, Letailleur M, Setif V, et al. Sperm chromatin condensation defects, but neither DNA fragmentation nor aneuploidy, are an independent predictor of clinical pregnancy after Intracytoplasmic Sperm Injection. J Assist Reprod Genet. 2019; https://doi.org/10.1007/s10815-019-01492.

4. Zhao Y, Kan FWK. Human OVGP1 enhances tyrosine phosphorylation of proteins in the fibrous sheath involving AKAP3 and increases sperm-zona binding. J Assist Reprod Genet. 2019; https:// doi.org/10.1007/s10815-019-01502.

Publisher's note Springer Nature remains neutral with regard to jurisdictional claims in published maps and institutional affiliations. 